\title{
An approach to collaboration through horizon scanning in the field of sclerochronology
}

\author{
Madelyn J. Mette', T. Trofimova² S.J. Alexandroff ${ }^{3}$ and E. Tray ${ }^{4}$
}

\section{Horizon scanning is an exercise which aims to collaboratively identify research priorities within a field. As exemplified by recent work led by members of the PAGES Early-Career Network in the field of sclerochronology, additional benefits include gained experience in collaboration, networking, and knowledge development.}

Defining top research priorities within a discipline is a common pursuit toward advancing the state of the art. An increasingly applied strategy, termed "horizon scanning", relies on community-based input and collaboration through surveys and rating systems to develop a combined perspective on important emerging topics and/or persistent challenges in the field (Sutherland et al. 2011). The concepts are then presented as research questions to be addressed within continuing and future work, providing a kind of roadmap for development of the field (for recent examples see Patiño et al. 2017; Sutherland et al. 2020).

Following this objective, four members of the PAGES Early-Career Network (authors of this article; PAGES ECN; pastglobalchanges. org/ecn), recently led a horizon-scanning exercise for the field of sclerochronology. This field encompasses the study of physical and chemical variations in the accretionary hard tissues of organisms, and the temporal context in which they formed. Physical and geochemical proxies from coral, bivalve, and otolith archives, for example, contribute to research questions across ecology, paleoclimatology, archaeology, and other fields. Sclerochronology has experienced significant growth over the past decade, with new methods and applications continually being explored. Because of this, we realized the need and an opportunity to formulate research priorities within our field.

Published as part of a special issue after the 5th International Sclerochronology Conference in Split, Croatia (ISC; June 2019), a manuscript by Trofimova et al. (2020) represents the first peer-reviewed scientific product by the PAGES ECN. The manuscript was strengthened by the significant involvement of 23 additional experts (and coauthors) in sclerochronology. The work can serve as a long-standing resource to be reassessed as the field develops. Completion of the project gives us the opportunity to reflect on the process and its impact on those involved. In this article, we provide a brief description of our process and scientific findings followed by discussion of some of the strategies we used and lessons learned in our approach to collaboration. We suggest that such horizonscanning projects can provide huge benefits to early-career researchers, especially by giving them increased visibility, allowing them to develop a better understanding of the subject area, and providing them with invaluable experience in international collaboration.

\section{Overall process}

The sclerochronology horizon-scanning exercise involved soliciting "high priority" research questions from the sclerochronology community, compiling and categorizing the questions, returning the list to the community in the form of a priority-ranking survey, and, ultimately, presenting the top 50 priority research questions alongside brief descriptions of their context and motivation (Fig. 1). The questions were divided into two broad categories: foundations and applications (Fig. 2). Foundations in sclerochronology include questions addressing knowledge gaps in our understanding of sclerochronological archives. This category was further divided into six subtopics. Applications encompass the use of sclerochronological techniques to address long-standing research questions in other fields. This category was divided into three subtopics. An extra category, Cutting-edge sclerochronology, comprised questions that expert coauthors deemed significant or uniquely important even though the community had not ranked them within the top 50 .

\section{Results}

While the field of sclerochronology has experienced rapid growth over the past few decades, the top priority questions ranked by the community reveal that there is still significant advancement to be made in building upon our foundational knowledge (i.e. the underlying basis for proxy application). For example, an emergent theme from the Foundations section was the need for a better understanding of the mechanisms behind biological control over biomineralization. Top questions also emphasized the establishment and widespread use of common standards for data management and analysis as an important strategy to enable future work. The large number of questions that were focused on applications, however, suggests the field is developed enough to provide new insights into important topics across the natural and social sciences. Top questions highlighted the precise dating and high resolution (at least annual) attainable from many sclerochronological archives as key advantages to solving long-standing questions in climate science and ecology, in particular. The entire list of highest-ranked questions recognizes the breadth of opportunity within the field of sclerochronology (Foundations and Applications), while also acknowledging novel applications that may have been overlooked in the ranking process (Cutting-edge sclerochronology).

\section{Strategies and lessons learned}

The triennial ISC provided a venue to discuss our idea with senior colleagues, gain commitment from collaborators, and establish processes moving forward. Project leaders and invited experts shared and debated feedback on our proposed goals and

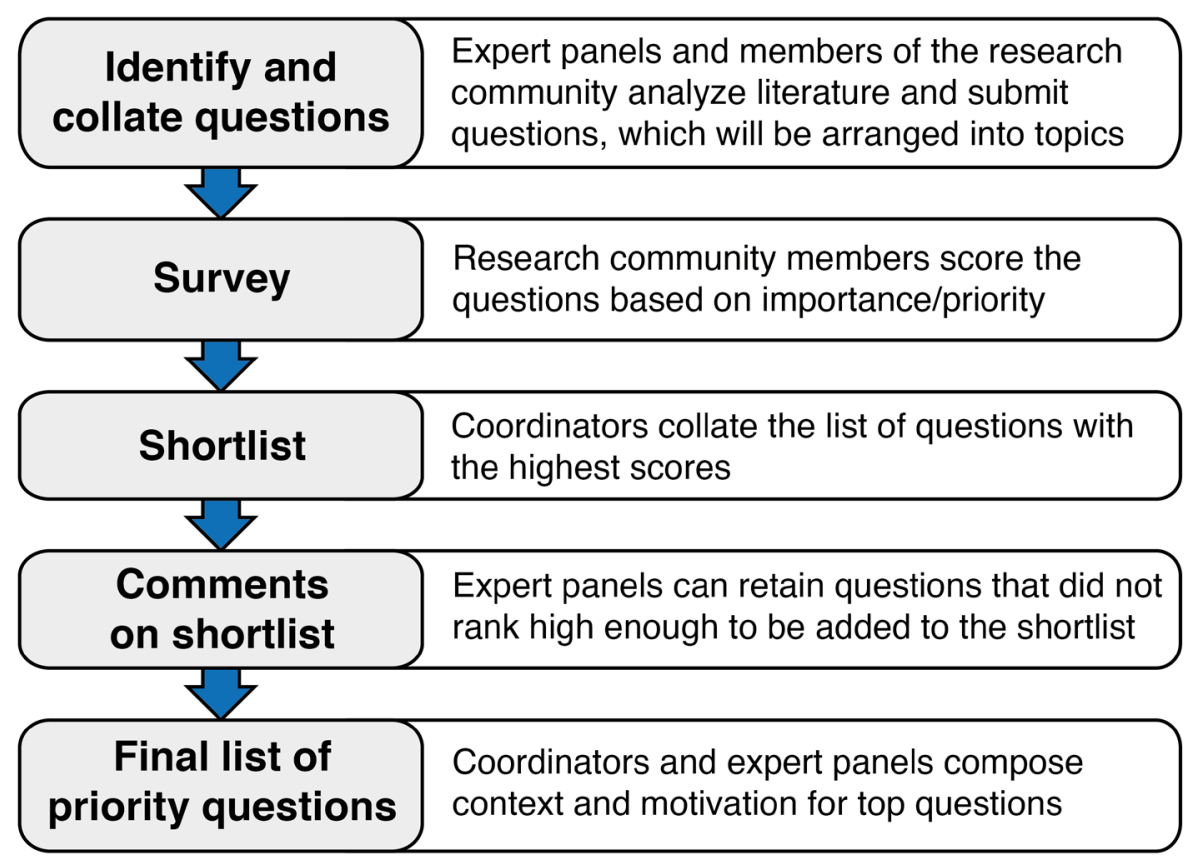

Figure 1: A brief overview of the horizon-scanning process in sclerochronology. 
Categories and topics of questions

\section{Foundations of sclerochronology}

$\rightarrow$ Biomineralization

$\rightarrow$ Drivers of skeletal growth

$\rightarrow$ Data standards

$\rightarrow$ Data analysis and interpretation

$\rightarrow$ Temperature reconstructions

$\rightarrow$ Archive-specific research

\section{Applications of sclerochronology}

$\rightarrow$ Global climate

$\rightarrow$ Paleoceanography

$\rightarrow$ Paleoecology and humanenvironmental interactions

\section{Cutting-edge sclerochronology}

Figure 2: Organization of categories and topics encompassing the horizon-scanning project in sclerochronology, spanning development across foundations and applications of the field. Additional questions retained by expert panels (Cutting-edge) were also presented.

approach. After the conference, the wider sclerochronology community was invited, via email list servers and social media, to submit research questions they deemed important. With 202 initial submissions, deciding how much editorial liberty we should exercise to arrive at a consistent format and use of terminology was a great challenge. Most suggestions required some reformatting in order to be presented on an equal playing field while still preserving their original intent. Providing more clear and strict guidelines during the question submission phase may have alleviated this challenge to some extent.

During our discussions with experts, alternative visions for the article were shared including the suggestion to present only a handful of broad research "themes" for future work. While we found these suggestions valuable, we were committed to following a traditional horizon-scanning model as exemplified in other fields (e.g. Seddon et al. 2014; Sutherland et al. 2020) as the first such exercise to be performed in sclerochronology. The final list of questions provides ideas for future work and can be pursued as either part or the whole of individual projects.
While research groups independently defining their own research agendas can certainly lead to innovation and progress, a unified research agenda provides an opportunity to cooperatively and more rapidly move the field forward (Sutherland and Woodruff 2009). Furthermore, presenting focused research questions to the community may encourage multiple, reproducible studies on the same subjects, which is essential for reaching scientific consensus. We ultimately received strong and continuous support in this effort throughout the project. Indeed because we as early-career researchers will inherit the future of the field, we were granted the freedom to follow our vision for contributing to that future.

The broad call to the scientific commu nity resulted in a bias toward some of the most commonly studied archives (bivalves, otoliths), well-represented regions (North Atlantic, Europe), and prevalent research applications (climate science, ecology) presented at the ISC conferences. While an effort was made to properly acknowledge and overcome this bias (see Trofimova et al. 2020 for further discussion), we believe that seeking out the involvement of research groups, more directly and from different fields and regions, could help improve representation across the diversity of sclerochronology, thus providing a more valuable result overall.

We resolved the challenges discussed above through successful international collaboration. Because it was unfeasible to hold virtual meetings with all or even most coauthors, communication to the project team occurred through email and file sharing. The lead authors were primarily responsible for man aging questions, discussions, and feedback on two to four subtopics each, followed by review or input on all other subtopics. The coauthors were assigned to expert panels that aligned with their research expertise and tasked with providing feedback on those subtopics. All coauthors had access to one shared document, stored in a cloud, and were given clear instructions on how to use online tools for adding content or providing feedback. This strategy was critical in keeping all authors involved and updated.

\section{Key insights}

The collaborative nature of horizon scanning offers an approach which allows earlycareer researchers, in particular, to significantly contribute to the future of a field. A meaningful byproduct of the exercise was increased visibility, collaborative experience, and knowledge development for those involved. After completion of our horizon scanning project in sclerochronology, we all felt more equipped to approach multiple subtopics within our field with confidence, having had the opportunity to lead in depth scientific discussions and help find a consensus among a community of experts. Few other research or training activities could have provided such a comprehensive and rigorous experience. While our project occurred before the COVID-19 pandemic, we recognize that horizon-scanning initiatives may represent prime opportunities to perform large collaborations without the requirement of in-person meetings. The PAGES ECN is well equipped to foster such collaborations through horizon-scanning projects, data compilations, review papers new research projects, and other pursuits that benefit from broad collaboration.

\section{AFFILIATIONS}

US Geological Survey, St. Petersburg Coastal and Marine Science Center, St. Petersburg, Florida, USA ${ }^{2}$ NORCE Norwegian Research Centre, Bjerknes Centre for Climate Research, Bergen, Norway

${ }^{3}$ College of Life and Environmental Sciences, University of Exeter, UK

"Marine and Freshwater Research Centre Galway-Mayo Institute of Technology, Ireland

\section{CONTACT}

Madelyn Mette: mmette@usgs.gov

REFERENCES

Patiño J et al. (2017) J Biogeogr 44: 963-983

Seddon A et al. (2014) J Ecol 102: 256-267

Sutherland W, Woodruff H (2009) Trends Ecol Evol 24: 537-527

Sutherland W et al. (2011) Methods Ecol Evol 2: 238-247 Sutherland W et al. (2020) Trends Ecol Evol 35: 81-90 Trofimova T et al. (2020) Estuar Coast Shelf Sci: 245 